\title{
A SHARP UPPER BOUND FOR DISCRETE INEQUALITIES OF GRONWALL-BELLMAN TYPE
}

\author{
E. Thandapani \\ Department of Mathematics \\ Madras University P.G. Centre \\ Salem-636 011, Tamil Nadu, India
}

\begin{abstract}
A sharp upper bound is given for solutions of a discrete inequality of the Gronwall-Bellman type. The bound, which is the exact solution of the corresponding discrete equation, is obtained by reducing the equation to a system of difference equations.
\end{abstract}

AMS Subject Classification: 39A99.

Key Words: Difference inequality, exact solution, difference equations.

\section{INTRODUCTION.}

It is well known that the discrete analog of Gronwall-Bellman lemma and its generalization, Bihari's lemma established by Jones [4] and Sugiyama [6] (also see [1], [2], [3], [5]), play an important role in the theory of difference equations and numerical analysis. They have been used, for example, to obtain upper bounds of solutions and in [7] it is shown that sharp upper bounds can be obtained by reducing the integral inequalities to a system of differential inequalities. The purpose of this note is to demonstrate how similar estimates can be derived for solutions of the discrete inequality

(1) $u(x) \leq b_{0}(x)+b_{1}(x) \sum_{j=0}^{n} \sum_{x_{1}=0}^{x-1} a_{0}\left(x_{1}\right) \sum_{x_{2}=0}^{x_{1}-1} a_{1}\left(x_{2}\right) \ldots \sum_{x_{j+1}=0}^{x_{j}-1} a_{j}\left(x_{j+1}\right) u\left(x_{j+1}\right)$

where $x_{0}=x$. When $n=1$, this inequality was considered by Pachpatte [5] for which he gave an upper bound for $u$ which is not sharp. Further,

\footnotetext{
${ }^{0}$ Received: December 1988; Revised: May 1989
} 
this type of inequality was considered by Agarwal and Thandapani [1] and their upper bound is also not sharp.

\section{MAIN RESULT.}

Here we shall obtain a sharp upper bound for solutions of (1). The procedure is to find the exact solution of the corresponding discrete equation by reducing it to a system of difference equations.

Let $N$ denote the set $\{0,1, \ldots\}$ and define the operator

$$
T_{k-1} u(x)=\sum_{j=k-1}^{n} \sum_{x_{k}=0}^{x-1} a_{k-1}\left(x_{k}\right) \ldots \sum_{x_{j+1}=0}^{x_{j}-1} a_{j}\left(x_{j+1}\right) u\left(x_{j+1}\right)
$$

with $x_{k-1}=x$ for each $k=1,2, \ldots, n+1$. It is easily seen that

$$
\Delta T_{k-1} u(x)=a_{k-1}(x)\left[u(x)+T_{k} u(x)\right] \quad k=1,2, \ldots, n+1
$$

where $\Delta$ is the operator defined by $\Delta z(x)=z(x+1)-z(x)$. Further, let $A$ denote the $n+1$ by $n+1$ matrix

$$
A(x)=\left[\begin{array}{cccccc}
1+b_{1} a_{0} & a_{0} & 0 & \ldots & 0 & 0 \\
b_{1} a_{1} & 1 & a_{1} & \ldots & 0 & 0 \\
\vdots & & & & & \\
b_{1} a_{n-1} & 0 & 0 & \ldots & 1 & a_{n-1} \\
b_{1} a_{n} & 0 & 0 & \ldots & 0 & 1
\end{array}\right]
$$

and $B$ the column vector

$$
B=\left[a_{0} b_{0}, a_{1} b_{0}, \ldots, a_{n} b_{0}\right]^{T}
$$

Theorem: Let $u, b_{0}, b_{1}$ and $a_{j}$ be real-valued, non-negative numbers defined on $N$, and $a_{j} \not \equiv 0, j=0,1, \ldots, n$. If $u$ satisfies (1), then for $x \in N$

$$
u(x) \leq b_{0}(x)+b_{1}(x) v_{1}(x)
$$

where $v_{1}$ is the first component of the $n+1$-vector function

$$
V(x)=\sum_{s=0}^{x-1} Y(x) Y^{-1}(s) B(s)
$$


and $Y(x)$ is the fundamental matrix satisfying

$$
Y(x+1)=A(x) Y(x) .
$$

Proof: With the operator (2) define

$$
v_{k}(x)=T_{k-1} u(x)
$$

and note that $v_{k}(0)=0, k=1,2, \ldots, n+1$. By (3) we have

$$
\begin{aligned}
\Delta v_{k}(x)=a_{k-1}(x)\left[u(x)+v_{k+1}(x)\right] \\
\leq a_{k-1}(x)\left[b_{0}(x)+b_{1}(x) v_{1}(x)+v_{k+1}(x)\right] \quad k=1,2, \ldots, n+1
\end{aligned}
$$

since $u(x) \leq b_{0}(x)+b_{1}(x) T_{0} u(x)=b_{0}(x)+b_{1}(x) v_{1}(x)$ and $v_{n+2} \equiv 0$. Thus treating $v_{1}, v_{2}, \ldots, v_{n+1}$ as components of an $n+1$-vector function $V$, the system (10) can be rewritten as

$$
V(x+1) \leq A(x) V(x)+B(x), \quad V(0)=0
$$

where $A$ and $B$ are defined by (4) and (5), respectively. $V(0)=0$ denotes the zero column vector. As is well known, the solution of the initial value problem

$$
V(x+1)=A(x) V(x)+B(x), \quad V(0)=0
$$

is given by (7).

It is clear that if equality holds in (1), then the same is true in the above derivation, so that $w=b_{0}+b_{1} v_{1}$ is the exact solution of the corresponding difference equation.

As a simple example, consider (1) when $n=2, b_{0}=b_{1}=a_{0}=a_{1}=1$ and $a_{2}=2$. The system (11) yields

$$
\left[\begin{array}{l}
v_{1}(x+1) \\
v_{2}(x+1) \\
v_{3}(x+1)
\end{array}\right] \leq\left[\begin{array}{lll}
2 & 1 & 0 \\
1 & 1 & 1 \\
2 & 0 & 1
\end{array}\right]\left[\begin{array}{l}
v_{1} \\
v_{2} \\
v_{3}
\end{array}\right]+\left[\begin{array}{l}
1 \\
1 \\
2
\end{array}\right]
$$

with $v_{1}(0)=v_{2}(0)=v_{3}(0)=0$ for which a fundamental matrix $Y(x)$ can be readily obtained. We find

$$
v_{1}(x)=\frac{2}{7} 3^{x}+\frac{5}{7} \cos \frac{\pi}{3} x+\frac{11}{21} \sqrt{3} \sin \frac{\pi}{3} x-1 .
$$


Hence we have $u \leq v_{1}(x)+1=w(x)$. This bound is sharp since $w(x)$ is the solution of the corresponding difference equation which is equivalent to the problem

$$
\begin{gathered}
w(x+3)-4 w(x+2)+4 w(x+1)-3 w(x)=0 \\
w(0)=1, \quad w(1)=2, \quad w(2)=3
\end{gathered}
$$

\section{REFERENCES.}

1. Agarwal, R.P. and Thandapani, E., On discrete generalizations of Gronwall's inequality, Bull. Inst. Math. Acad. Sinica, 9 (1981), pp. 235-248.

2. Coffman, C.V., Asymptotic behaviour of solutions of ordinary difference equation, Trans. Amer. Math. Soc., 110 (1964), pp. 22-51.

3. Henrici, P., Discrete variable methods in ordinary differential equations, Wiley, New York (1962).

4. Jones, G.S., Fundamental Inequalities for discrete and discontinuous functional equations, SIAM J. Appl. Math., 12 (1964), pp. 43-47.

5. Pachpatte, B.G., Finite Difference inequalities and their applications, Proc. Nat. Acad. Sci. India, 43(A) (1973), pp 348-356.

6. Sugiyama, S., On the stability problems of difference equations, Bull. Sci. Eng. Res. Lab. Waseda Univ., 45 (1969), pp. 140-144.

7. Young, E.C., On integral inequalities of Gronwall-Bellman type, Proc. Amer. Math. Soc., 94 (1985), pp. 636-640. 


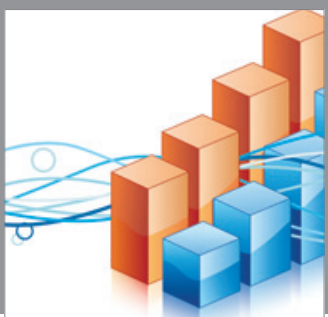

Advances in

Operations Research

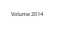

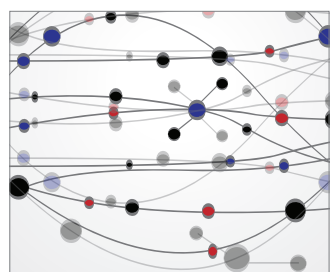

\section{The Scientific} World Journal
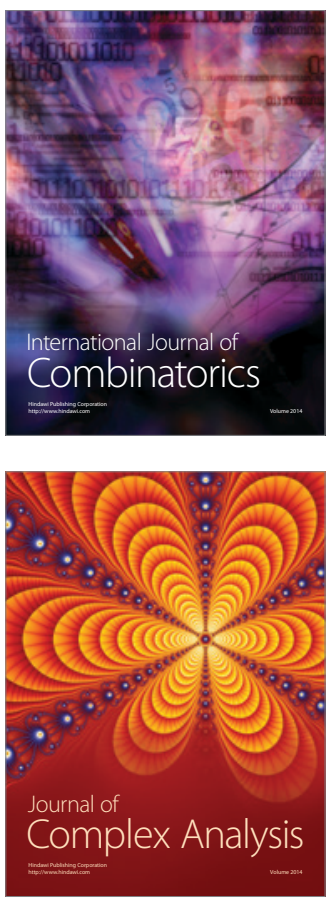

International Journal of

Mathematics and

Mathematical

Sciences
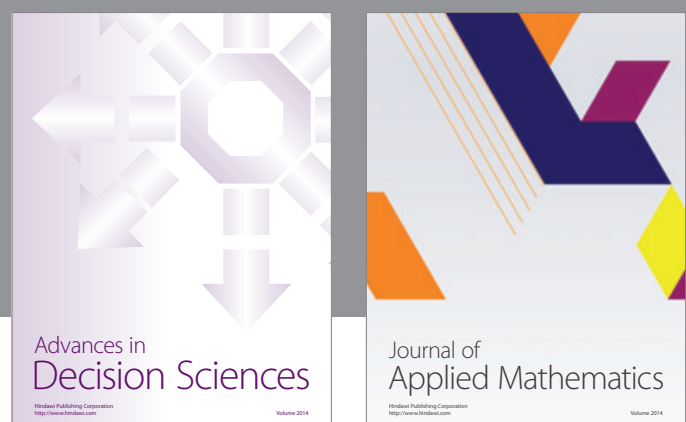

Journal of

Applied Mathematics
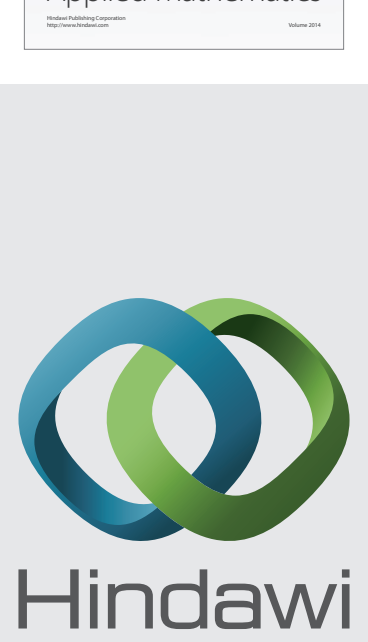

Submit your manuscripts at http://www.hindawi.com
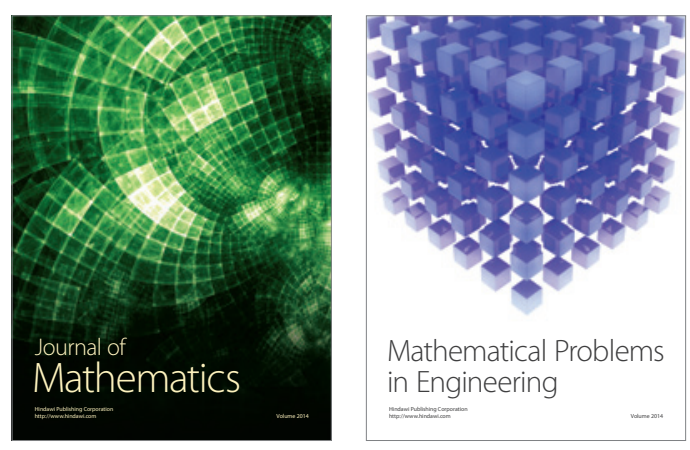

Mathematical Problems in Engineering
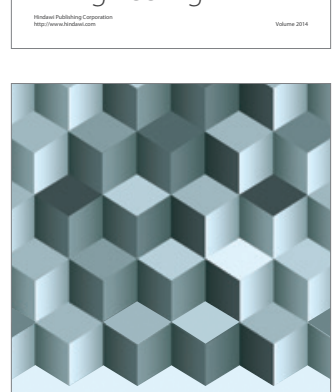

Journal of

Function Spaces
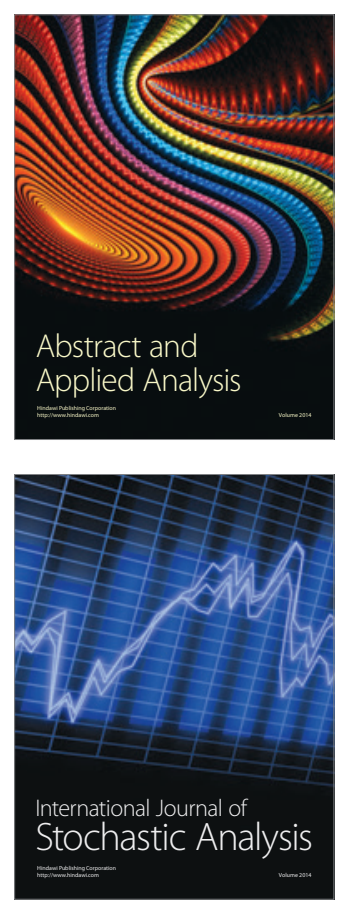

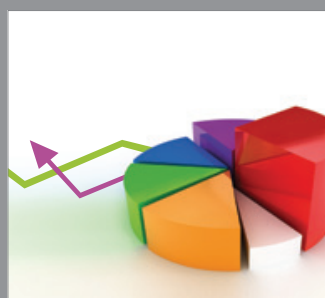

ournal of

Probability and Statistics

Promensencen
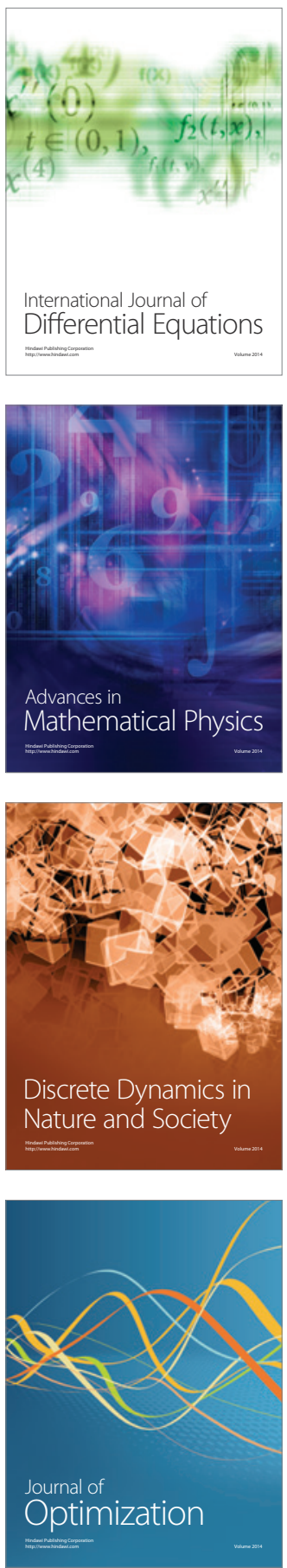